\title{
HEAT TRANSFER IN THE THERMAL ENTRANCE REGION OF A FLAT DUCT
}

\author{
A. S. JONES \\ (Received 4 February 1975)
}

\begin{abstract}
The problem of heat transfer in a duct or tube for large values of the Péclet number has traditionally been solved by assuming that diffusion in the axial direction is negligible. This approach was used by Graetz [2] for the circular tube and by Prins et al [5] for the flat duct to obtain a series solution for downstream temperature field.

Since these series converge very slowly in the neighbourhood of the origin, some other approach is necessary in the thermal entrance region. This was supplied by Lévêque [3] and extended by Mercer [4] who matched the Lévêque solution to the eigenfunction expansion.

In all these solutions it was assumed that the axial diffusion of heat was negligible, but this assumption is invalid close to the discontinuity, since in this region the axial temperature gradient is large and the fluid velocity is small, so that axial diffusion plays an important role.

In this paper, the assumptions implicit in Lévêque's solution are re-examined, and the correct approximation in the neighbourhood of the discontinuity as well as the solution which matches this into Lévêque's solution are presented. In the first of these solutions, diffusion is the only heat-transfer mechanism, while in the matching solution diffusion and convection are in balance.

The corresponding solutions for the case of prescribed flux on the boundary are also considered.
\end{abstract}

\section{Introduction}

If we consider the forced convection of heat in a tube or duct in which there is a sudden change of temperature in the wall, we see that there must also be a rapid axial change in temperature in the fluid adjacent to the wall. Furthermore, because of the no-slip boundary condition on fluid flows, the fluid velocity adjacent to the wall is low. This means that in this region at least, the diffusion of heat in the axial direction is important. 
The effects of axial diffusion on the solution can be investigated in two ways. The first, which has recently been adopted in many papers, is to determine the overall solution of the diffusion-convection equation retaining the term corresponding to axial diffusion. While this leads to a solution theoretically valid throughout the fluid, it retains the difficulty inherent in the Graetz solution that convergence of the series solution is slow in the thermal entrance region.

The alternative approach, which is adopted in this paper, is to consider a series of local approximations, none of which retain all the terms of the diffusion-convection equation, but which together provide an accurate representation of the thermal field. Since these solutions are of a simpler form than the general solution, they are more amenable to calculation, and as well they indicate the regions in which the various heat transfer mechanisms are relevant.

In this paper we consider heat transfer in a two-dimensional flat duct. The results however apply equally well to tubes with smooth cross-sections since the effects of curvature are negligible to the orders of magnitude considered here. Two cases are considered. The 'isothermal' case where the wall temperature is specified, and the 'adiabatic' case where we have insulated walls and point heat sources on the boundaries.

\section{Mathematical model}

We consider a flat duct with sides at $\eta=0$ and $\eta=a$ through which is flowing a fluid in fully developed steady laminar Poiseuille flow. Ignoring viscous dissipation, and assuming that the physical properties of the fluid are independent of the temperature, the diffusion-convection equation for the temperature $\theta(\xi, \eta)$ of the fluid is

$$
\kappa\left(\frac{\partial^{2} \theta}{\partial \xi^{2}}+\frac{\partial^{2} \theta}{\partial \eta^{2}}\right)=6 \frac{u_{m}}{a^{2}} \eta(a-\eta) \frac{\partial \theta}{\partial \xi}
$$

where $\kappa$ is the thermal diffusivity, $u_{m}$ is the mean fluid velocity and $\xi$ is the axial co-ordinate.

For the 'isothermal' case, the boundary conditions are

$$
\begin{array}{rlrl}
\theta(\xi, 0) & =\theta(\xi, a)=\theta_{0}, & \xi<0 ; \\
& =\theta_{1}, \quad \xi>0 . \\
\theta(\xi, \eta) \rightarrow \theta_{0}, & \xi \rightarrow-\infty & \\
\rightarrow \theta_{1}, & \xi \rightarrow+\infty .
\end{array}
$$


This corresponds to an incoming fluid, initially having the same temperature as the walls, which encounters a step change in wall temperature at the origin $\xi=0$.

For the 'adiabatic' case, the boundary conditions are

$$
\begin{aligned}
\theta_{\eta}(\xi, 0) & =-\phi \delta(\xi) \\
\theta_{\eta}(\xi, a) & =\phi \delta(\xi) \\
\theta(\xi, \eta) & \rightarrow \theta_{0}, \quad \xi \rightarrow-\infty \\
\theta(\xi, \eta) & \rightarrow \theta_{f}, \quad \xi \rightarrow \infty
\end{aligned}
$$

where $\theta_{f}$ is determined by considering the heat balance. This gives $\theta_{f}=$ $\theta_{0}+2 \kappa \phi / a u_{m}$.

This corresponds to forced convection in an insulated duct with point sources of heat on the walls at the origin.

Equation 2.1 is rendered non-dimensional by setting $x=\xi / a, y=\eta / a$ and introducing the Péclet number $P=6 u_{m} a / \kappa$, which is assumed to be large. Simultaneously we scale the temperature by setting

$$
T=\left(\theta-\theta_{0}\right) /\left(\theta_{1}-\theta_{0}\right) \quad \text { in the isothermal case }
$$

and

$$
T=\left(\theta-\theta_{0}\right) / \phi \quad \text { in the adiabatic case. }
$$

This yields the equation

$$
T_{x x}+T_{y y}=P y(1-y) T_{x},
$$

with boundary conditions

$$
\begin{array}{rlrl}
T(x, 0) & =T(x, 1)=0 & x<0 ; \\
& =1 \quad x>0 . \\
T(x, y) \rightarrow 0 & x \rightarrow-\infty ; \\
\rightarrow 1 & x \rightarrow \infty,
\end{array}
$$

for the isothermal case and

$$
\begin{aligned}
& T_{y}(x, 0)=-\delta(x), \\
& T_{y}(x, 1)=\delta(x), \\
& T(x, y) \rightarrow 0 \quad x \rightarrow-\infty, \\
& T(x, y) \rightarrow 12 / P \quad x \rightarrow \infty,
\end{aligned}
$$

for the adiabatic case. 


\section{The isothermal case}

(a) The Lévêque solution.

If we set $x=P X$, equation 2.2 reduces to

$$
P^{-2} T_{x x}+T_{y y}=y(1-y) T_{x},
$$

and the Graetz approximation is now obtained by ignoring the term $P^{-2} T_{X X}$. The resulting equation is further simplified near $y=0$ if we ignore the term $y^{2} T_{x}$ in comparison with $y T_{x}$. This gives the Lévêque approximation.

$$
T_{y y}=y T_{x} .
$$

One important consequence of the Graetz approximation is that it converts the differential equation from elliptic to parabolic type, so that the solution for $x<0$ is $T \equiv 0$.

The solution of 3.1 for $x>0$ is obtained by putting $\eta=y X^{-1 / 3}$, and assuming $T=f(\eta)$. This gives

$$
f^{\prime \prime}+\frac{1}{3} \eta^{2} f^{\prime}=0
$$

where $f(0)=1$ and $f \rightarrow 0$ as $\eta \rightarrow \infty$.

The solution is

$$
T=1-\int_{0}^{\eta} e^{-s^{3 / 9}} d s / \int_{0}^{\infty} e^{-s^{3 / 9}} d s
$$

which for small $\boldsymbol{\eta}$ is approximately

$$
\begin{aligned}
T & \simeq 1-\frac{3^{1 / 3}}{\Gamma(1 / 3)} y X^{-1 / 3} \\
& =1-\frac{(3 P)^{1 / 3}}{\Gamma(1 / 3)} y x^{-1 / 3} .
\end{aligned}
$$

In order to determine the region in which the Lévêque solution is relevant, it is necessary to rederive equation 3.1 in a more systematic fashion.

If we set $x=P^{-p} X, y=P^{-q} Y$ in equation 2.2, we obtain

$$
T_{Y Y}+P^{2(p-q)} T_{X X}=P^{1-3 q-p} Y T_{X}-P^{1-4 q+p} Y^{2} T_{X}
$$

The Lévêque equation $T_{Y Y}=Y T_{X}$ is therefore a valid approximation provided

and

$$
\begin{array}{r}
1-3 q+p=0 \\
2(p-q)<0
\end{array}
$$




$$
1-4 q+p<0,
$$

that is: $p=3 q-1, p<q$ and $q>0$.

These restrictions are satisfied if $0<q<\frac{1}{2}$ and $-1<p<\frac{1}{2}$. As $q \rightarrow 0$, $p \rightarrow-1$, the form of the equation becomes

$$
T_{Y Y}=\left(Y-Y^{2}\right) T_{X} \quad \text { i.e. the Graetz equation. }
$$

This means that the Lévêque solution matches into the Graetz solution when $y$ is $O(1)$ and $x$ is $O(P)$.

On the other hand, as $q \rightarrow \frac{1}{2}, p \rightarrow \frac{1}{2}$, the equation becomes

$$
T_{X X}+T_{Y Y}=Y T_{X},
$$

so that the effects of axial conduction should be included when $x$ is $O\left(P^{-1 / 2}\right)$ and $y$ is $O\left(P^{-1 / 2}\right)$.

It should be noted that

$$
\eta=Y X^{-1 / 3}=P^{q-p / 3} y x^{-1 / 3}=P^{1 / 3} y x^{-1 / 3},
$$

so that the Lévêque solution does not depend on the choice of $p, q$ in the relevant range.

From this analysis, we see that the Lévêque solution is valid in a region extending from close to the origin up to the region where the full Graetz solution applies. This agrees with Mercer's results, where calculations based on the first four perturbations of the Lévêque solution gave excellent agreement with those made using the Graetz solution.

(b) The approximation near the origin.

Near the origin, diffusion is the important heat transfer mechanism. Since the fluid is isotropic, we adopt equal scaling for both the $x$ and $y$ variables.

Setting $p=q$ in 3.2 , we obtain

$$
T_{X X}+T_{Y Y}=P^{1-2 p} Y T_{X}-P^{1-3 p} Y^{2} T_{X} .
$$

For $p>\frac{1}{2}$, this gives the approximation

$$
T_{X X}+T_{Y Y}=0
$$

and the solution which satisfies

$$
\begin{aligned}
T(X, 0) & =0, & & X<0, \\
& =1, & & X>0,
\end{aligned}
$$

and is bounded as $\left(X^{2}+Y^{2}\right) \rightarrow \infty$, is

$$
T=1-\frac{1}{\pi} \arctan (Y / X)=1-\frac{1}{\pi} \arctan (y / x),
$$


where the determination of $\arctan$ is chosen to make $T$ continuous; i.e. $0 \leqq \arctan Z \leqq \pi$.

In this region convection plays a negligible role in the heat transfer process.

As $p \rightarrow \frac{1}{2}$, we obtain again the equation

$$
T_{X X}+T_{Y Y}=Y T_{X}
$$

which serves to link the diffusion solution with Lévêque's solution.

(c) The intermediate solution: heat transfer in linear shear flow.

In the intermediate zone between the diffusion region at the origin and the Lévêque boundary layer, we have a region in which diffusion in both the axial and transverse directions is balanced by convection.

The boundary conditions applicable to equation 3.3 are

$$
\begin{aligned}
T(X, 0) & =0, & & X<0 ; \\
& =1, & & X>0 . \\
T(X, y) & \rightarrow 0, & & X \rightarrow-\infty ; \\
& \rightarrow 1, & & X \rightarrow+\infty ; \\
& \rightarrow 0, & & Y \rightarrow+\infty .
\end{aligned}
$$

To obtain the solution, we take the two-sided Laplace transform of 3.3 with respect to $X$.

If

$$
\begin{aligned}
\bar{T}(p, Y) & =\int_{-\infty}^{\infty} e^{-p X} T(X, Y) d X, \text { then } \\
\bar{T}_{Y Y} & =\left(p Y-p^{2}\right) \vec{T} \\
\bar{T}(p, 0) & =1 / p \\
\bar{T}(p, Y) & \rightarrow 0, \quad Y \rightarrow \infty .
\end{aligned}
$$

The solution of 4.1 which satisfies the boundary conditions is

$$
\bar{T}=\operatorname{Ai}\left(p^{1 / 3}(Y-p)\right) / p \operatorname{Ai}\left(-p^{4 / 3}\right)
$$

where $\operatorname{Ai}(z)$ is the Airy function.

The inversion of 4.2 is complicated by the presence of a branch point at $p=0$. If we introduce a branch cut from $-\infty$ to 0 along the negative real $p$ axis, we find that $\arg \left(p^{1 / 3}(Y-p)\right)$ and $\arg \left(-p^{4 / 3}\right)$ vary from $-\pi / 3$ to $7 \pi / 3$ as $\arg p$ varies from $-\pi$ to $\pi$. 
The zeros of $\operatorname{Ai}(z)$ occur when $\arg z=(2 n+1) \pi$, so that the poles of $\bar{T}$ occur when $p=0$ and at those points on the positive real axis for which

$$
p^{4 / 3}=a_{s}, \quad \text { where } \quad-a_{s} \text { is the } s \text { th zero of } \operatorname{Ai}(z) .
$$

Now

$$
T(X, Y)=\frac{1}{2 \pi i} \int_{c-\infty \infty}^{c+\infty} \frac{e^{p X}}{p} \frac{\operatorname{Ai}\left(p^{1 / 3}(Y-p)\right)}{\operatorname{Ai}\left(-p^{4 / 3}\right)} d p,
$$

where $0<c<a_{1}^{3 / 4}$ in order to ensure convergence of $\int_{-\infty}^{\infty} e^{-p X} T(X, 0) d X$ and to ensure that $T \rightarrow 0, X \rightarrow-\infty$.

For $X<0$, we can close the path of integration on the right to obtain

$$
T(X, Y)=\sum_{s=1}^{\infty} \frac{3}{4} \cdot \frac{\exp \left(\left(a_{s}\right)^{3 / 4} X\right) \mathrm{Ai}\left(a_{s}^{1 / 4} Y-a_{s}\right)}{a_{s} \mathrm{Ai}^{\prime}\left(-a_{s}\right)} .
$$

For $X>0$, we deform the contour until it runs from $-\infty$ to 0 and back along each side of the branch cut, bearing in mind the pole, residue 1 , at $p=0$. This -gives

$$
\begin{gathered}
T(X, Y)=1+\frac{1}{2 \pi i} \int_{0}^{\infty} \frac{e^{-s x}}{s} \frac{\operatorname{Ai}\left(s^{1 / 3}(Y+s) e^{i \pi / 3}\right)}{\operatorname{Ai}\left(s^{4 / 3} e^{i \pi / 3}\right)} d s \\
-\frac{1}{2 \pi i} \int_{0}^{\infty} \frac{e^{-s X}}{s} \frac{\operatorname{Ai}\left(s^{1 / 3}(Y+s) e^{-i \pi / 3}\right.}{\operatorname{Ai}\left(s^{4 / 3} e^{-i \pi / 3}\right)} d s \\
=1-\frac{1}{\pi} \int_{0}^{\infty} \frac{e^{-s X}}{s} \frac{\mathrm{Ai}\left(-s^{1 / 3}(Y+s)\right) \operatorname{Bi}\left(-s^{4 / 3}\right)-\operatorname{Bi}\left(-s^{1 / 3}(Y+s)\right) \operatorname{Ai}\left(-s^{4 / 3}\right)}{\operatorname{Ai}^{2}\left(-s^{4 / 3}\right)+\mathrm{Bi}^{2}\left(-s^{4 / 3}\right)} d s,
\end{gathered}
$$

where $\operatorname{Bi}(z)$ is the second solution of the Airy equation, and extensive use has been made of the formula

$$
\operatorname{Ai}\left(z e^{ \pm 2 \pi / 3}\right)=\frac{1}{2} e^{\pi i / 3}[\mathrm{Ai}(z) \mp i \operatorname{Bi}(z)] .
$$

For large $X(>0)$ the asymptotic form of $T(X, Y)$ is obtained by expanding the non-exponential part of the integral in powers of $s$.

This gives

$$
\begin{aligned}
T(X, Y) & \sim 1-\frac{1}{\pi} \int_{0}^{\infty} \frac{e^{-s x}}{s}\left(\frac{\sqrt{ } 3}{2} \cdot \frac{3^{1 / 3} \Gamma(2 / 3)}{\Gamma(1 / 3)} s^{1 / 3} Y\right) d s \\
& =1-\frac{1}{\pi} \sin \frac{\pi}{3} \cdot \frac{3^{1 / 3} \Gamma(2 / 3)}{\Gamma(1 / 3)} \Gamma(1 / 3) \cdot Y X^{-1 / 3} \\
& =1-\frac{3^{1 / 3}}{\Gamma(1 / 3)} Y X^{-1 / 3} \\
& =1-\frac{3^{1 / 3} P^{1 / 3}}{\Gamma(1 / 3)} y x^{1 / 3} .
\end{aligned}
$$


Hence for large $X$, this solution matches with the Lévêque solution.

For small $X(>0)$ we employ the polar form of the Airy functions

$$
\begin{aligned}
& \mathrm{Ai}(-z)=M(z) \cos \theta(z) \\
& \mathrm{Bi}(-z)=M(z) \sin \theta(z) .
\end{aligned}
$$

Hence

$$
T(X, Y)=1-\frac{1}{\pi} \int_{0}^{\infty} \frac{e^{-s X}}{s} \frac{M\left(s^{1 / 3}(Y+s)\right)}{M\left(s^{4 / 3}\right)} \cdot \sin \left\{\left(\theta\left(s^{4 / 3}\right)-\theta\left(s^{1 / 3}(Y+s)\right)\right\} d s .\right.
$$

Setting $t=s X$ we have

$$
\begin{aligned}
T(X, Y)= & 1-\frac{1}{\pi} \int_{0}^{\infty} \frac{e^{-t}}{t} \frac{M\left((t / X)^{4 / 3}(1+X Y / t)\right)}{M\left((t / X)^{4 / 3}\right)} \cdot \sin \left\{\theta\left((t / X)^{4 / 3}\right)\right. \\
& \left.-\theta\left((t / X)^{4 / 3}\left(1+\frac{X Y}{t}\right)\right)\right\} d t \\
= & 1-\frac{1}{\pi} \int_{0}^{\delta}() d t-\frac{1}{\pi} \int_{\delta}^{\infty}() d t .
\end{aligned}
$$

Since the integrand behaves like $C \cdot t^{-2 / 3} Y X^{-1 / 3}$ in the neighbourhood of $t=0$, the first integral is $0\left(\delta^{1 / 3}\right)$ provided $Y X^{-1 / 3}$ remains bounded.

In the second integral we now consider $X, Y \ll \delta$, subject to the above provision.

For large $Z$,

and

$$
M(Z) \sim \frac{1}{\sqrt{ } \pi} Z^{-1 / 4}
$$

$$
\theta(Z) \sim \frac{\pi}{4}-\frac{2}{3} Z^{3 / 2}
$$

Hence the second integral

$$
\begin{aligned}
& -\frac{1}{\pi} \int_{\delta}^{\infty} \frac{e^{-t}}{t}(1+X Y / t)^{-1 / 4} \cdot \sin \left(\frac{2}{3}\left(\frac{t}{X}\right)^{2}\left(1+\frac{X Y}{t}\right)^{3 / 2}\right. \\
& \left.-\frac{2}{3}\left(\frac{t}{X}\right)^{2}\right) d t \\
& -\frac{1}{\pi} \int_{\delta}^{\infty} \frac{e^{-t}}{t} \sin \left(t \frac{Y}{X}\right) d t \\
& -\frac{1}{\pi} \int_{0}^{\infty} \frac{e^{-t}}{t} \sin \left(t \frac{Y}{X}\right) d t \\
= & -\frac{1}{\pi} \arctan (Y / X) .
\end{aligned}
$$


Consequently, for small $X, Y$,

$$
\begin{aligned}
T(X, Y) & \sim 1-\frac{1}{\pi} \arctan (Y / X) \\
& =1-\frac{1}{\pi} \arctan (y / x)
\end{aligned}
$$

so that this solution also matches into the diffusion solution near the origin.

In the upstream region we have the power series representation for $T(X, Y)$. For large $|X|$ this gives

$$
\begin{aligned}
T(X, Y) & \sim 3 \exp \left(a_{1}^{3 / 4} X\right) \operatorname{Ai}\left(a_{1}^{1 / 4} Y-a_{1}\right) /\left\{4 a_{1} \mathrm{Ai}^{\prime}\left(-a_{1}\right)\right\} \\
& \sim e^{189 X} \operatorname{Ai}(1.24 Y-2.34) / 2.1826
\end{aligned}
$$

which has a maximum value of $.245 e^{1.89 x}$ when $Y=1.07$.

This approximation gives $T<.01$ for $X<-1.7$, so that significant preheating of the fluid is confined to a region of radius $O\left(P^{1 / 2}\right)$ of the origin, and the upstream temperature decays exponentially as $P^{1 / 2}$.

For small $|X|$, the series converges slowly, which makes approximations difficult. However, if we consider $T_{Y}(X, 0)$ we have

$$
T_{Y}(X, 0)=\frac{3}{4} \sum_{1}^{\infty} \exp \left(a_{s}^{3 / 4} X\right) / a_{s}^{3 / 4},
$$

and $\left(a_{s}\right)^{3 / 4} \sim \sqrt{ }(3 \pi s / 2)$, so that for small $X$,

$$
T_{Y}(X, 0) \sim \frac{3}{4} \int_{0}^{\infty} d s \exp \left(3 \pi s x^{2} / 2\right)^{1 / 2} /(3 \pi s / 2)^{1 / 2}=-1 / \pi X,
$$

which agrees with the derivative of $1-(1 / \pi) \arctan (Y / X)$, offering an indirect proof that the upstream solution also matches the diffusion solution.

The behaviour of the solution near the origin is illustrated in Figure 1, which shows some isotherms for the shear flow solution.

\section{The adiabatic case}

The scaling analysis which determines the regions of interest for the isothermal case carries over unchanged for the adiabatic case. Hence we will once again have a diffusion region close to the origin, a shear region around this, a Lévêque boundary layer region and a Graetz region far downstream.

Some care is needed in computing the boundary conditions since they are affected by the scaling.

If $x=P^{-p} X$, and $y=P^{-q} Y$, then $\partial T / \partial Y=P^{-q} \partial T / \partial y$, and $\delta(x)=$ $P^{p} \delta(X)$. 


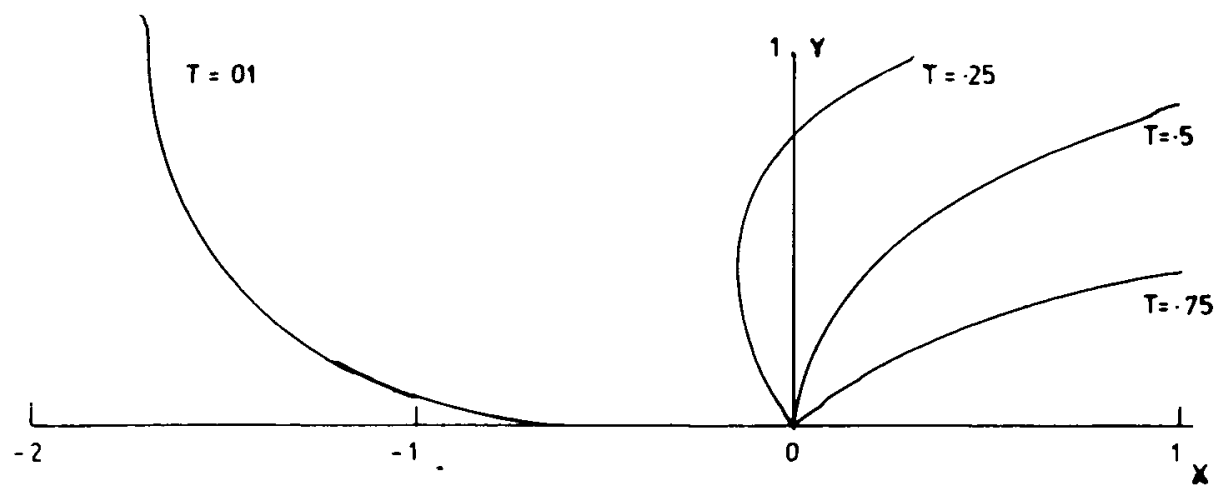

Figure 1. Isotherms in the neighbourhood of the origin.

Therefore the boundary condition

$$
\frac{\partial T}{\partial y}=-\delta(x)
$$

becomes

$$
\frac{\partial T}{\partial Y}=P^{p-a} \delta(X)
$$

In the diffusion and shear regions $p=q$ so that the form of the boundary condition is unchanged, but in the Lévêque region $p=3 q-1$, giving

$$
\frac{\partial T}{\partial Y}=P^{2 q-1} \delta(X)
$$

and in the Graetz region $p=-1, q=0$, so that $\partial T / \partial Y=P^{-1} \delta(X)$.

In the diffusion region we have

$$
\begin{aligned}
T_{X X}+T_{Y Y} & =0, \\
T_{Y}(X, 0) & =-\delta(X),
\end{aligned}
$$

for which the solution which grows least at infinity is

$$
\begin{aligned}
T(X, Y) & =-\frac{1}{\pi} \log V\left(X^{2}+Y^{2}\right)+c \\
& =-\frac{1}{\pi} \log \left(R / R_{0}\right),
\end{aligned}
$$

where the constant $R_{0}$ is indeterminate. 
In the shear region we have

$$
T_{X X}+T_{Y Y}=Y T_{X}
$$

with boundary conditions

$$
\begin{array}{rlrl}
T_{Y}(X, 0) & =-\delta(X) \\
T(X, Y) & \rightarrow 0 & X \rightarrow-\infty \\
& \rightarrow 0 & Y \rightarrow \infty .
\end{array}
$$

Using the same method of solution as before, we obtain

$$
T=\frac{3}{4} \sum_{1}^{\infty} \frac{\exp \left(\left(a_{s}^{1}\right)^{3 / 4} X\right) \mathrm{Ai}\left(\left(a_{s}^{1}\right)^{1 / 4} Y-a_{s}^{1}\right)}{\left(a_{s}^{1}\right)^{3 / 2} \mathrm{Ai}\left(-a_{s}^{1}\right)}
$$

for $X<0$, where $-a_{s}^{1}$ is the $s$ th zero of the derivative $\operatorname{Ai}^{\prime}(Z)$ of $\operatorname{Ai}(Z)$, and

$$
T=\frac{1}{\pi} \int_{0}^{\infty} d S \frac{e^{-S x}}{S^{1 / 3}} \frac{\mathrm{Ai}\left(-S^{1 / 3}(Y+S)\right) \mathrm{Bi}^{\prime}\left(-S^{4 / 3}\right)-\mathrm{Ai}^{\prime}\left(-S^{4 / 3}\right) \mathrm{Bi}\left(-S^{1 / 3}(Y+S)\right)}{\left.\left(\mathrm{Ai}^{\prime}\left(-S^{4 / 3}\right)\right)^{2}+\mathrm{Bi}^{\prime}\left(-S^{4 / 3}\right)\right)^{2}}
$$

for $X>0$.

For large $X(>0)$,

$$
\begin{aligned}
T(X, Y) & \sim \frac{1}{\pi} \int_{0}^{\infty} d s \frac{e^{-s x}}{S^{1 / 3}} \cdot\left(\frac{\Gamma(1 / 3) \sin (\pi / 3)}{3^{1 / 3} \Gamma(2 / 3)}\right) \\
& =3^{-1 / 3} X^{-2 / 3} / \Gamma(2 / 3) \\
& =(3 P)^{-1 / 3} x^{-2 / 3} / \Gamma(2 / 3) .
\end{aligned}
$$

For small $X(>0)$ the behaviour of $T$ is more difficult to determine.

Once again we use the polar representations of the Airy functions. In this case it gives

$$
T(X, Y)=\frac{1}{\pi} \int_{0}^{\infty} \frac{e^{-s x}}{S^{1 / 3}} \cdot \frac{M\left(S^{1 / 3}(Y+S)\right)}{N\left(S^{4 / 3}\right)} \cdot \sin \left\{\phi\left(S^{4 / 3}\right)-\theta\left(S^{1 / 3}(Y+S)\right)\right\} d s
$$

where $N$ and $\phi$ are the modulus and amplitude of the derived functions.

Splitting this integral to give $T(X, Y)=(1 / \pi)\left(\int_{0}^{\kappa}+\int_{\kappa}^{\infty}\right)() d s$, the first integral is $O\left(\kappa^{2 / 3}\right)$ since the integrand is $O\left(S^{-1 / 3}\right)$ at the origin.

In the second integral we set $t=S X$ to give

$$
\begin{aligned}
I_{2}=\frac{1}{\pi} \int_{\alpha X}^{\infty} X^{1 / 3} \frac{e^{-t} M\left((t / X)^{4 / 3}(1+X Y) / t\right)}{N\left((t / X)^{4 / 3}\right)} \sin \left\{\left(\phi(t / X)^{4 / 3}\right)\right. & \\
& \left.-\theta\left((t / X)^{4 / 3}(1+X Y / t)\right)\right\} \frac{d t}{X}
\end{aligned}
$$


Now assuming $\kappa$ is large enough to justify the asymptotic forms for $M$ and $N$, we obtain

$$
\begin{aligned}
I_{2} \sim & \frac{1}{\pi} \int_{\kappa X}^{\infty} d t X^{-2 / 3} \frac{e^{-t}}{t^{1 / 3}} \frac{\pi^{-1 / 2}\left((t / X)^{-1 / 3}(1+X Y / t)^{-1 / 4}\right)}{\pi^{-1 / 3}(t / X)^{1 / 3}} \\
& \times \sin \left(\frac{3 \pi}{4}-\frac{2}{3} \frac{t^{2}}{x^{2}}-\frac{\pi}{4}+\frac{2}{3} \frac{t^{2}}{X^{2}}(1+X Y / t)^{3 / 2}\right) \\
= & \frac{1}{\pi} \int_{\kappa X}^{\infty} d t\left(e^{-t} / t\right)(1+X Y / t)^{-1 / 4} \cos \left(\frac{2}{3} t^{2} / X^{2}\left((1+X Y / t)^{3 / 2}-1\right)\right) \\
\sim & \frac{1}{\pi} \int_{\kappa X}^{\infty} d t\left(e^{-t} / t\right) \cos (t Y / X) \\
= & \left.\frac{1}{\pi} e^{-t} \cos (t Y / X) \log t\right]_{\kappa X}^{\infty} \\
& +\frac{1}{\pi} \int_{\kappa X}^{\infty} d t e^{-t} \log t(\cos (t Y / X)+Y / X \sin (t Y / X)) \\
\sim & -\frac{1}{\pi} \log \kappa X \\
& +\frac{1}{\pi} \int_{0}^{\infty} d t e^{-t} \log t(\cos (t Y / X)+Y / X \sin (t Y / X)) \quad \text { as } X, Y \rightarrow 0 \\
= & -\frac{1}{\pi} \log \kappa X-\frac{1}{\pi} \log \left(\gamma \vee\left(1+Y^{2} / X^{2}\right)\right) \\
= & -\frac{1}{\pi} \log V\left(X^{2}+Y^{2}\right)-\frac{1}{\pi} \log (\kappa \gamma) .
\end{aligned}
$$

Hence $T(X, Y) \sim-(1 / \pi) \log \sqrt{ }\left(X^{2}+Y^{2}\right)+c$, which agrees with the solution in the diffusion region, but gives no analytic information regarding the indeterminate constant.

A numerical value for the constant can however be obtained by considering $T(X, 0)$ for $X<0$. The sum

$$
T(X, 0)=\frac{3}{4} \sum_{1}^{\infty} \exp \left(p_{s} X\right) / p_{s}^{2} \quad \text { where } p_{s}=\left(a_{s}^{1}\right)^{3 / 4}
$$

converges slowly for small $X$ since $p_{s}$ is $O\left(S^{1 / 2}\right)$, as we would expect from the logarithmic singularity in $T$ when $X=0$. This limits the accuracy to which the calculations can be performed. However the values computed give $T=$ $-\log (|X| / 2.8)$ for $-.1<X<-.02$.

Hence near the origin we have 


$$
T \simeq-\frac{1}{\pi} \log \left(\left(\frac{P^{1 / 2}}{2.8}\right)\left(x^{2}+y^{2}\right)^{1 / 2}\right) .
$$

The boundary layer approximation is given by the solution of

$$
T_{Y Y}=Y T_{X}
$$

subject to the boundary conditions

$$
\begin{aligned}
T_{Y}(X, 0) & =-P^{2 q-1} \delta(X) \\
T(0, Y) & =0 \\
t & \rightarrow 0, \quad Y \rightarrow \infty .
\end{aligned}
$$

Using the normal Laplace transform we obtain

$$
\bar{T}_{Y Y}=p Y \bar{T}
$$

with

$$
\bar{T}_{Y}=-P^{2 q-1} \text { when } \quad Y=0,
$$

so that

$$
\bar{T}(p, Y)=3^{1 / 3} \Gamma(1 / 3) P^{2 q-1} \operatorname{Ai}\left(p^{1 / 3} Y\right) / p^{1 / 3}
$$

the inverse of which is

$$
\begin{aligned}
T(X, Y) & =3^{-1 / 3} P^{2 q-1} X^{-2 / 3} \exp \left(-Y^{3} / 9 X\right) / \Gamma(2 / 3) \\
& =(3 P)^{-1 / 3} x^{-2 / 3} \exp \left(-P y^{3} / 9 x\right) / \Gamma(2 / 3)
\end{aligned}
$$

which matches into the shear flow solution.

The downstream "Graetz" solution is usually given in terms slightly of different boundary conditions (Cess and Shaffer [1]) involving a constant boundary flux. However, the modification for the boundary conditions used here is straightforward, and the solution is

$$
T(X, Y)=\frac{12}{P}+\sum_{n=1}^{\infty} c_{n}^{\prime} Y_{n}(\xi) \exp \left(-16 \beta_{n}^{2} X\right)
$$

whère $\xi=2\left(Y-\frac{1}{2}\right)$ and the eigenvalues and eigenfunctions are those calculated by Cess and Shaffer [1].

\section{Summary}

From the above analysis we can see that there are four regions of physical significance for forced convection at large Péclet number. To these may be 
added the core region in which the fluid temperature is essentially unchanged from its upstream value.

In the region close to the origin, where $x, y$ are $O\left(P^{-1 / 2-z}\right)$ convection is negligible and we obtain a simple diffusion solution. Since there is no convective term in the equation, we have an essentially symmetric temperature field in the neighbourhood of the origin. This region encompasses the singularities of the solution, and in the isothermal case we have an infinite amount of heat transferred through this region.

Surrounding this region is the shear flow region in which the effects of convection first become apparent. All significant upstream diffusion takes place in this region, with the temperature decaying exponentially as $P^{1 / 2} x$ as $x$ tends to $-\infty$, with less than one percent of the heat escaping further than a distance $O\left(P^{-1 / 2}\right)$ upstream of the origin. Downstream the heat is confined to a region close to the wall and the solution matches into the boundary layer solution. The effects of diffusion in the axial direction are confined to these two regions so that axial diffusion is significant only in a region of radius $O\left(P^{-1 / 2}\right)$ near the origin.

The third region is the boundary layer adjacent to the wall downstream of the origin. In this region, as in the first two, the temperature field is not affected by either curvature effects or by the presence of a second wall, so that to all intents and purposes we have forced convection from a flat plate. This is especially noticeable in the adiabatic case, where in the absence of a second boundary the shear and boundary layer solutions tend to zero far downstream. However, when $x$ is $O(P)$ the solutions are $O\left(P^{-1}\right)$ as they should be for the matching into the downstream solution.

In the final downstream region the solution takes on the character of the tube or duct in which the heat transfer is taking place. In the two dimensional case treated here this corresponds to the interaction of the two boundary layers, while in other cases there are also curvature effects to be taken into account. It should be noted that an analysis of the case of a circular tube shows that there is no intermediate region in which curvature effects on the boundary layer are significant.

For small values of the Peclet number all these regions coalesce to a certain extent and we have a significant region of upstream heat penetration. However the diffusion region close to the origin and the downstream region retain their character; the former because it represents a region in which convection is not important, an effect which is enhanced as the Péclet number becomes smaller, and the latter because it is a region in which the temperature field varies only slowly in the axial direction, the difference being the rate at which the temperature approaches its final value. 


\section{References}

[1] R. D. Cess and E. C. Shaffer, 'Heat transfer to laminar flow between parallel plates with a prescribed wall heat flux', Appl. Sci. Res. (A) 8, 5 (1959), 339-344.

[2] L. Graetz, 'Über der warmeleitungsfährigkerten der flussigkeiten', Ann. Phys. 18 (1883), 79-94.

[3] A. Lévêque, 'Résolution théorique du problème de l'échange de chaleur par circulation d'un fluide visqueux en mouvement tranquille a l'intérieur d'un tube cylindrique', Comptes Rendus 185 (1927), 1190-1192.

[4] A. McD. Mercer, 'The growth of the thermal boundary layers in laminar flow between parallel fiat plates', Appl. Sci. Res. (A) 8, 5 (1959), 357-365.

[5] J. A. Prins, J. Mulder and J. Schenk, 'Heat transfer in laminar flow between parallel plates', Appl. Sci. Res. (A) 2, 5/6 (1951), 431-438.

Department of Mathematics,

University of Queensland,

St Lucia, Qld 4067,

Australia. 